\title{
A superposed epoch analysis of auroral evolution during substorm growth, onset and recovery: open magnetic flux control of substorm intensity
}

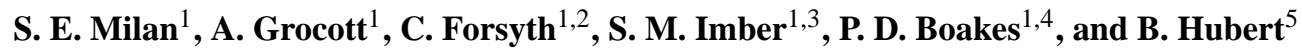 \\ ${ }^{1}$ Department of Physics and Astronomy, University of Leicester, Leicester, UK \\ ${ }^{2}$ Mullard Space Sciences Laboratory, University College London, Surrey, UK \\ ${ }^{3}$ Heliophysics Division, NASA GSFC, Greenbelt, MD, USA \\ ${ }^{4}$ British Antarctic Survey, Natural Environment Research Council, High Cross, Cambridge, UK \\ ${ }^{5}$ Laboratory of Planetary and Atmospheric Physics, University of Liege, Liege, Belgium
}

Received: 31 July 2008 - Revised: 16 December 2008 - Accepted: 22 January 2009 - Published: 11 February 2009

\begin{abstract}
We perform two superposed epoch analyses of the auroral evolution during substorms using the FUV instrument on the Imager for Magnetopause-to-Aurora Global Explorer (IMAGE) spacecraft. The larger of the two studies includes nearly 2000 substorms. We subdivide the substorms by onset latitude, a measure of the open magnetic flux in the magnetosphere, and determine average auroral images before and after substorm onset, for both electron and proton aurora. Our results indicate that substorms are more intense in terms of auroral brightness when the open flux content of the magnetosphere is larger, and that magnetic flux closure is more significant. The increase in auroral brightness at onset is larger for electrons than protons. We also show that there is a dawn-dusk offset in the location of the electron and proton aurora that mirrors the relative locations of the region 1 and region 2 current systems. Superposed epoch analyses of the solar wind, interplanetary magnetic field, and geomagnetic indices for the substorms under study indicate that dayside reconnection is expected to occur at a faster rate prior to low latitude onsets, but also that the ring current is enhanced for these events.
\end{abstract}

Keywords. Magnetospheric physics (Auroral phenomena; Magnetospheric configuration and dynamics; Storms and substorms)

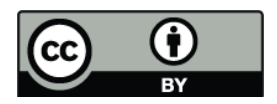

Correspondence to: S. E. Milan (steve.milan@ion.le.ac.uk)

\section{Introduction}

Since its discovery in the 1960s (e.g. Akasofu, 1964) the auroral substorm has been the subject of intense study due to the recognition that it plays a key role in solar windmagnetosphere-ionosphere coupling. In this paper we investigate the evolution of the aurora during the growth, expansion, and recovery phases of substorms in a quantitative, statistical fashion. Previous workers have undertaken similar studies, for instance Mende et al. (2003), Coumans et al. (2007) and Gjerloev et al. (2007), providing much insight into the average characteristics of the auroral evolution during substorms. However, our primary focus in this paper is to examine the role of the substorm in solar windmagnetosphere coupling, specifically in the closure of open flux previously accumulated through magnetic reconnection at the dayside magnetopause. Hence, our statistics concentrate on the substorm response to differing amounts of open flux in the magnetosphere at substorm onset. In addition, whereas previous studies have examined a limited number of events, usually of the order of 100 , our study encompasses observations from nearly 2000 substorms.

As proposed by Dungey (1961) magnetic reconnection between the interplanetary magnetic field (IMF) and the terrestrial magnetopause results in an open magnetosphere, with open field lines being stretched antisunwards to form the magnetotail, before being pinched off again by reconnection in the neutral sheet of the tail. Dungey originally envisaged the resulting circulation of field and plasma, now known as the Dungey cycle, as a steady state phenomenon. However, it became apparent that this could not be the case, that the nightside reconnection rate could not anticipate changes in

Published by Copernicus Publications on behalf of the European Geosciences Union. 
the dayside reconnection rate, and hence the level of interconnection between the magnetosphere and the solar wind must be variable. This lead to the formulation of what is now known as the expanding/contracting polar cap paradigm, or ECPC, which explains how variable rates of dayside and nightside reconnection lead to changes in the size of the polar cap and excite magnetospheric and ionospheric convection (Siscoe and Huang, 1985; Cowley and Lockwood, 1992). An extension of this was the explicit suggestion that substorms were the process by which the Dungey cycle was closed in the magnetotail (Lockwood and Cowley, 1992).

In this picture the bright auroral ovals encircle the dim polar caps, which themselves map to the magnetotail lobes comprised of open magnetic field lines. As the fraction of magnetospheric flux that is open increases the polar caps expand and the auroral ovals move to lower latitudes; conversely a contraction of the polar caps signifies a closure of open flux by reconnection in the tail (e.g. Milan et al., 2003, 2007). The auroral evolution during substorms can be understood within this framework. The equatorward progression of the auroral ovals during substorm growth phase (McPherron, 1970) indicates the increase in open flux through dayside reconnection. As the open flux increases the tail magnetopause flares outwards increasing the cross-section presented to the flow of the solar wind and hence the pressure inside. At some point this pressure increase can no longer be sustained and reconnection is triggered in the magnetotail neutral sheet to reduce the amount of open flux. Associated with this is the onset of substorm auroral break-up and the substorm expansion phase, as the auroral bulge progresses polewards into the polar cap, eastwards and westwards, marking the expanding region of newly-closed flux. Recovery phase follows as the magnetosphere returns to a quiescent state.

It has been suggested that the amount of open magnetic flux present in the magnetosphere at the time of substorm onset determines the intensity of the substorm, that is the auroral brightness and/or the strength of the auroral electrojets (e.g. Akasofu, 1975; Kamide et al., 1999), though there is some debate as to what causes a particular substorm to trigger at a particular open flux level (e.g. Milan et al., 2008). The aim of the present study is to provide a quantitative estimate of the average auroral evolution during substorms, particularly concentrating on differences due to the open flux content of the magnetosphere at onset. Two studies are undertaken to investigate this. In the first, reported in Sect. 2, we perform a superposed epoch analysis of the characteristics of 41 substorms observed during a 10 day period first investigated by Milan et al. (2008). In the second study, reported in Sect. 3, we perform a superposed epoch analysis of auroral images taken by the Imager for Magnetopause-toAurora Global Exploration (IMAGE) mission, keyed to a list of the onset times of some 2000 substorms compiled by Frey et al. (2004). We discuss our findings in Sect. 4.
The IMAGE mission provided almost 5 years of observations of the Northern and Southern Hemisphere UV aurora, between 2000 and 2005, using the Far Ultraviolet (FUV) instrument (Mende et al., 2000a, b) which comprised the Wideband Imaging Camera (WIC) and the Spectrographic Imager (SI). WIC was sensitive to auroral emissions over a broad range of the UV spectrum, the primary component of which is associated with precipitating electrons. The SI12 channel imaged Doppler-shifted Lyman $\alpha$ emission associated with precipitating protons. The orbital geometry and spin of the IMAGE spacecraft was such that images were captured with a cadence of $2 \mathrm{~min}$ for approximately $10 \mathrm{~h}$ of the $14 \mathrm{~h}$ orbit. In this paper we use observations from both WIC and SI12 to investigate the auroral evolution during substorms.

\section{Superposed epoch analysis of Milan et al. (2008) ob- servations}

Milan et al. (2008) analysed 10 days, 28 August to 6 September 2005, of WIC auroral observations from the IMAGE spacecraft to determine variations in the open flux content of the magnetosphere related to changes in upstream solar wind and IMF conditions and the occurrence of substorms. The open flux threading the ionospheric polar cap, $F_{P C}$, was determined by integrating the radial component of an assumed dipolar magnetic field through the dim region encircled by the auroral oval (see also Boakes et al., 2008); that is the poleward boundary of the aurora was identified with the open/closed field line boundary (OCB). As described by Milan et al. (2003), SuperDARN radar observations and precipitating-particle measurements from polarorbiting DMSP satellites were used to constrain the location of the OCB, where available. It is difficult to determine the uncertainty in values of $F_{P C}$ determined in this way, but Milan et al. (2003) suggested that an over- or underestimation of the location of the OCB by $1^{\circ}$ of latitude at all local times would lead to an under- or overestimation of $F_{P C}$ by approximately $10 \%$.

49 substorms were identified during the 10-day period, of which 41 had accompanying auroral images. One of the main conclusions of that study was that the open flux content of the magnetosphere increased dramatically and substorms became more intense during geomagnetic storm activity when the SYM-H index was depressed. Here we conduct a superposed epoch analysis of $F_{P C}$, the maximum in the nightside auroral intensity, $I_{\max }$, the AL and AU auroral electrojet indices, and the SYM-H index, and the predicted dayside reconnection rate for the 41 substorms identified by Milan et al. (2008). The predicted dayside reconnection rate, $\Phi_{D}$, is the Kan and Lee (1979) reconnection electric field multiplied by the effective width of the solar wind channel that reconnects with the dayside magnetopause. This reconnection potential is related to upstream solar wind and IMF conditions by

$\Phi_{D}=L_{\mathrm{eff}} V_{S W} B_{Y Z} \sin ^{2} \frac{1}{2} \theta$, 
where $V_{S W}$ is the solar wind speed, $B_{Y Z}$ is the transverse component of the IMF, i.e. $B_{Y Z}=\sqrt{B_{Y}^{2}+B_{Z}^{2}}, \theta$ is the IMF clock angle and $L_{\text {eff }}=2.75 R_{E}$ (Milan et al., 2008).

Epoch time $t$ is centred on substorm onset which was identified in most cases as an increase in auroral brightness, a decrease in $F_{P C}$ associated with magnetotail reconnection, and a substorm bay in AL. To investigate the influence of varying amounts of open flux in the system at substorm onset we have divided the substorms into four categories:

(i) $F_{P C}(t=0)<0.45 \mathrm{GWb}$,

(ii) $0.45<F_{P C}(0)<0.55 \mathrm{GWb}$,

(iii) $0.55<F_{P C}(0)<0.65 \mathrm{GWb}$,

(iv) $F_{P C}(0)>0.65 \mathrm{GWb}$.

These limits were chosen so that there were similar numbers of substorms in each category, that is $9,13,9$, and 10, respectively. Epoch time runs from two hours before to two hours after onset and is divided into 6-min bins. The results are shown in Fig. 1. In each panel the thick solid line shows the evolution of the median of the parameter in question, while the thin lines show the upper and lower quartiles in each bin.

We find that approximately one hour prior to substorm expansion phase onset there is an increase in the open flux of the magnetosphere: the substorm growth phase. Accompanying this growth phase is a modest increase in the auroral brightness. In most cases, at expansion phase onset there is a more dramatic increase in brightness, the open flux content decreases, and there is a negative excursion in the AL index. The exception is category $i$ in which a decrease in open flux is observed following onset, but there is no corresponding increase in brightness or bay in AL.

Overall, the observations clearly show that as the open flux at onset increases there is a dramatic increase in: (1) the auroral brightening associated with the substorm; (2) the growth of open flux prior to onset; (3) the decrease in open flux after onset; (4) the duration of the decrease in open flux; and (5) the magnitude of the AL bay associated with the substorm. Substorms in category i close approximately $0.1 \mathrm{GWb}$ of flux, whereas in category iv this rises to 0.3 to $0.4 \mathrm{GWb}$. A similar change is seen in the amount of open flux accumulated during the growth phase. In each category the growth phase lasts approximately one hour. On the other hand, the duration of the decrease in flux after onset increases from $30 \mathrm{~min}$ in the case of category i to $80 \mathrm{~min}$ in the case of category iv. In category $i$, the minimum in median $\mathrm{AL}$ reaches approximately $-200 \mathrm{nT}$, increasing to $-600 \mathrm{nT}$ in category iv.

The dayside reconnection voltage prior to onset increases from category i to category iv, from an average of $20 \mathrm{kV}$ to an average of $100 \mathrm{kV}$, explaining the more dramatic accumulation of open flux during the growth phase in the last category. Lastly, we note that SYM-H becomes more negative

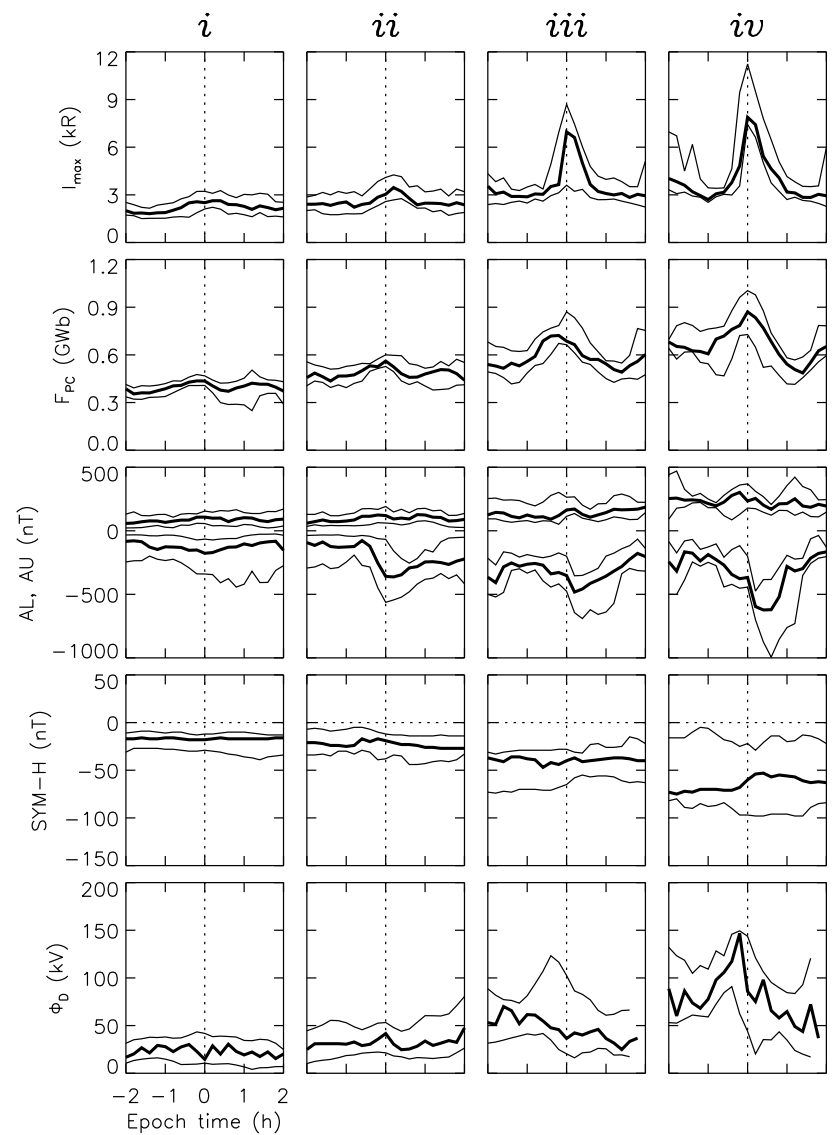

Fig. 1. Superposed epoch analysis of 41 substorms identified by Milan et al. (2008). From top to bottom, the parameters are: the maximum nightside auroral intensity $I_{\max }$ as measured by WIC; the open magnetic flux $F_{P C}$ estimated from the size of the polar cap; the AL and AU auroral indices; the SYM-H index; the estimated dayside reconnection rate $\Phi_{D}$. The substorms have been categorized by the open flux content of the magnetosphere at substorm onset. Thick and thin lines show the evolution of the median and upper and lower quartile values as a function of epoch time, centred on substorm onset.

from category i to iv, indicating that $F_{P C}$ increases to larger values prior to substorm onset when the ring current is enhanced.

Examination of the relationship between the median and quartiles of, say, $I_{\max }$ and $\Phi_{D}$, suggests that there are perhaps sudden changes in these parameters within categories iii and iv. However, we feel that a much larger sample of events would be needed to ascertain whether this effect is real.

\section{Superposed epoch analysis of IMAGE observations}

To build on these results, we performed a superposed epoch analysis of the auroral evolution during substorms for as large a sample of substorms as possible, though concentrating on observations of the Northern Hemisphere auroral oval. 

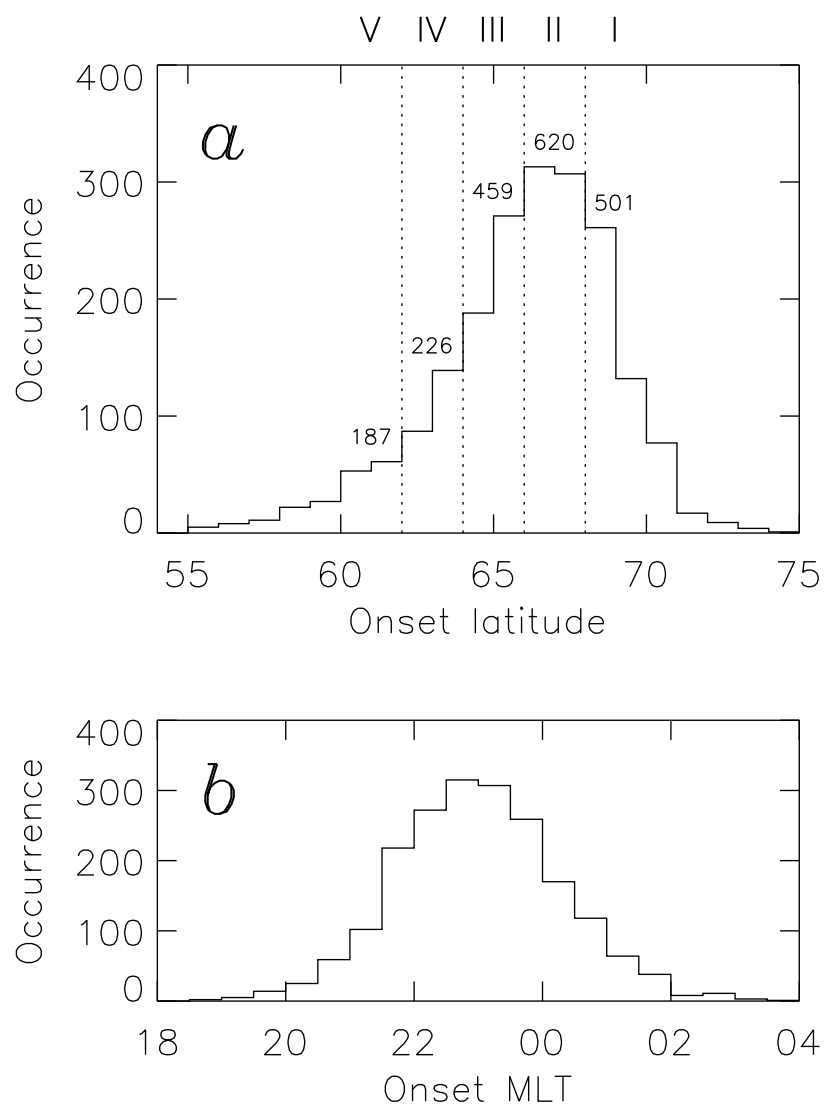

Fig. 2. (a) The distribution of onset latitudes for the 1993 substorms identified by Frey et al. (2004) during the period of our study. Vertical dotted lines identify the onset latitude categorizes I to V; numbers indicate the number of substorms in each category. (b) The magnetic local time distribution of onsets.

Precession of the apogee of the orbit of IMAGE meant that the Northern Hemisphere auroral oval was imaged best during the first two years of the mission, so we focussed our study on the 2-year period May 2000 to April 2002. Due to contamination of the WIC observations by dayglow we limited study of WIC images to the winter months of November, December, and January. Our primary dataset thus comprised close to 400000 images from SI12 and 100000 images from WIC. This dataset was pre-processed to speed up subsequent analysis. Each image was mapped into a magnetic latitude and MLT coordinate system and then re-binned into a $40 \times 40$ Cartesian grid of cells $222 \times 222 \mathrm{~km}$ in size $(222 \mathrm{~km}$ is equivalent to $2^{\circ}$ of latitude), centred on the geomagnetic pole with rows and columns aligned along the noon-midnight and dawn-dusk meridians.

Substorm onset timings were taken from the study of Frey et al. (2004), who defined onset as a prolonged brightening of the nightside auroral oval in the IMAGE dataset. Three criteria were used to indentify onsets: (1) a clear local brightening of the aurora had to occur; (2) the aurora had to expand to the poleward boundary of the auroral oval and spread azimuthally in local time for at least $20 \mathrm{~min}$; (3) $30 \mathrm{~min}$ had to pass since the previous onset. Ideally, this study covered the first two years of the IMAGE mission, identifying 1993 substorms during our period of interest. Frey et al. (2004) recorded a number of characteristic features of each substorm onset, including the magnetic latitude, $\Lambda_{\text {onset }}$, and magnetic local time (MLT) of the initial onset brightening. Onsets were observed at magnetic latitudes between $55^{\circ}$ and $74^{\circ}$; Fig. 2a presents the occurrence distribution of onset latitudes for our period of interest. Onsets at high latitudes indicate substorms occurring on a contracted auroral oval, onsets at low latitudes on an expanded oval, with corresponding variations in the amount of open flux in the magnetosphere. Averaging all of these substorms together would inevitably hide interesting dynamics and so we sub-divided our dataset into five categories by substorm onset latitude, $\Lambda_{\text {onset }}$, which we defined to be:

(I) $\Lambda_{\text {onset }}>68^{\circ}$,

(II) $66^{\circ}<\Lambda_{\text {onset }}<68^{\circ}$,

(III) $64^{\circ}<\Lambda_{\text {onset }}<66^{\circ}$,

(IV) $62^{\circ}<\Lambda_{\text {onset }}<64^{\circ}$,

(V) $\Lambda_{\text {onset }}<62^{\circ}$,

as indicated in Fig. 2a, where the number of onsets in each category is also shown. For completeness we also show the MLT distribution of onsets in Fig. 2b. Most onsets were observed between 21:00 and 01:00 MLT, as also found by Gjerloev et al. (2007). We examined the MLT distribution of onsets within each of our five latitudinal bins and found that none had a significant deviation from the overall MLT distribution.

From this it was straightforward to produce a superposed epoch analysis of auroral evolution keyed to onset time. We chose our epochs to run from one hour prior to onset to two hours after onset, and divided these three hours into 1810 min bins. Then we averaged the observed intensity in each of the $40 \times 40$ cells in all pre-processed images that fell within each category, resulting in 10 time-series of images, one for each latitude category and camera. By the way of example we show selected images from two of these time-series, the category IV onsets for both WIC and SI12, in Fig. 3. The selected images are from the time bins centred on $t=-55,-5$, +15 , and +115 min from substorm onset. To guide the eye, superimposed on each image is a $K_{P}=3$ statistical oval from Feldstein and Starkov (1967), calculated from the functional representation of Holzworth and Meng (1975).

We note that there is a considerable dawn-dusk asymmetry in the WIC and SI12 observations: the intensity of the auroral oval is greatest at dusk as observed by SI12 and greatest at dawn as observed by WIC. As will be discussed in more detail later, there is also an offset in the location of the WIC 


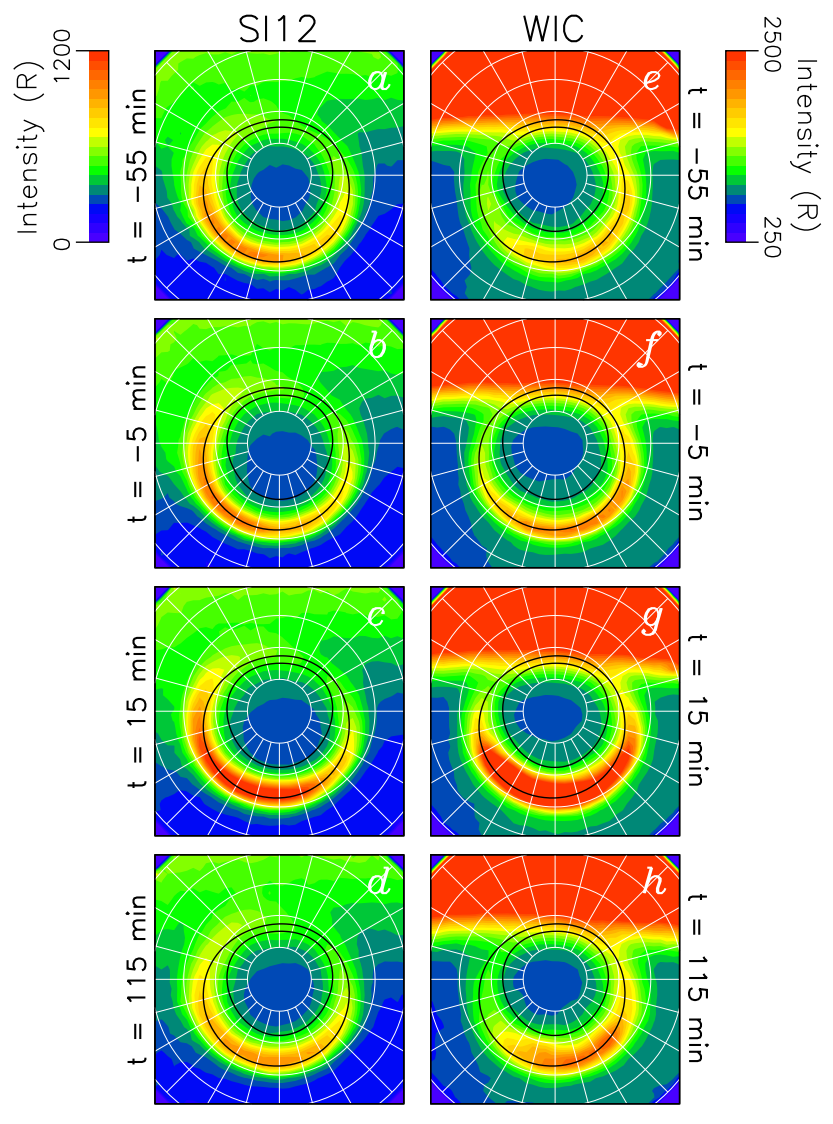

Fig. 3. A selection of SI12 and WIC average auroral images for category IV substorms. The data are presented in a polar geomagnetic latitude and MLT frame, with 12:00 MLT to the top of each image. Radial lines indicate MLT meridians and concentric circles are lines of constant geomagnetic latitude in steps of $10^{\circ}$. Superimposed on each image is a $K_{P}=3$ Feldstein and Starkov (1967) average auroral oval.

and SI12 ovals along the dawn-dusk meridian, SI12 being shifted duskwards with respect to WIC. The SI12 and WIC ovals move to lower latitudes between $t=-55$ and $-5 \mathrm{~min}$, most apparent in the midnight sector, as the polar cap expands during the growth phase. There is a marked increase in auroral brightness and the formation of an auroral bulge by $t=15 \mathrm{~min}$. By $t=115 \mathrm{~min}$ the auroral oval has returned to a configuration similar to that at $t=-55 \mathrm{~min}$, though there is still some residual excess brightness in the post-midnight sector, especially in the WIC observations.

We extract further information from each averaged image by determining the latitude of the peak intensity along the dawn, dusk, noon and midnight meridians. The method by which this is achieved is shown in Fig. 4, for the auroral image of Fig. 3c. The noon-midnight and dawn-dusk profiles of the image are shown in Fig. 4a and c, respectively, by crosses. The vertical dotted lines represent the location of the geomagnetic pole. Each of the four meridians, from low latitude to the pole, are treated separately. Following the ap-
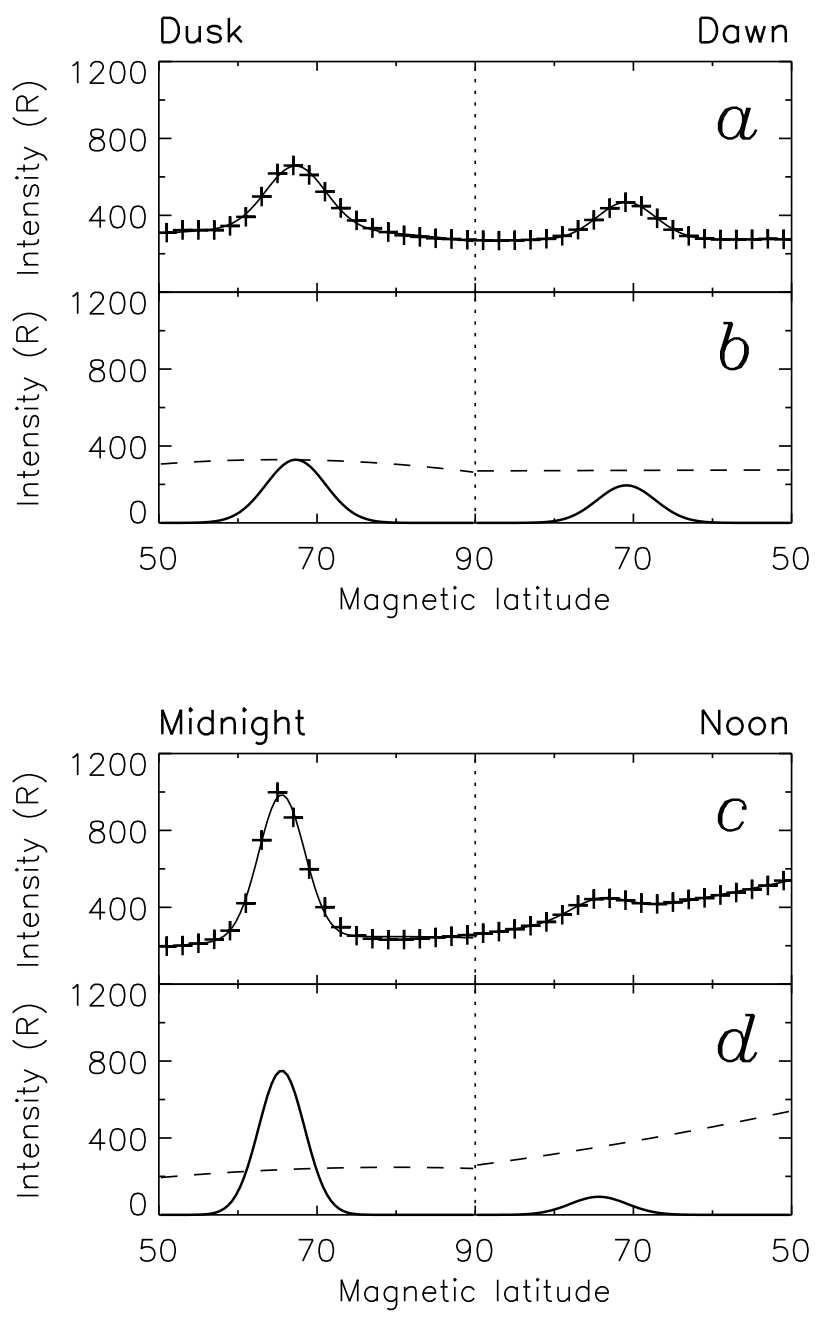

Fig. 4. Illustrative of the fitting technique used to find the Gaussian auroral profile (solid curves) and a polynomial background (dashed curves) corresponding to the raw average intensity profiles (crosses) along the dawn-dusk and noon-midnight meridians of an SI12 image.

proach of Carbary et al. (2003), the background intensity and the auroral contribution are assumed to have a polynomial and Gaussian profile, respectively, such that the variation of image intensity $I$ with magnetic latitude $\Lambda$ can be described by

$$
I(\Lambda)=a \Lambda^{2}+b \Lambda+c+A_{\max } \exp \left\{-\left(\frac{\Lambda-\Lambda_{\max }}{\Delta \Lambda}\right)^{2}\right\} .
$$

Here $a, b, c$ are constants describing the background profile and $A_{\max }, \Lambda_{\max }$ and $\Delta \Lambda$ describe the auroral contribution, being the peak in intensity, the latitude of the peak, and the width of the profile, respectively. All six constants are determined by a least-squares fitting procedure. The fit to each profile is shown in Fig. $4 \mathrm{a}$ and $\mathrm{c}$ by solid curves, indicating a good representation of the data. The polynomial background 

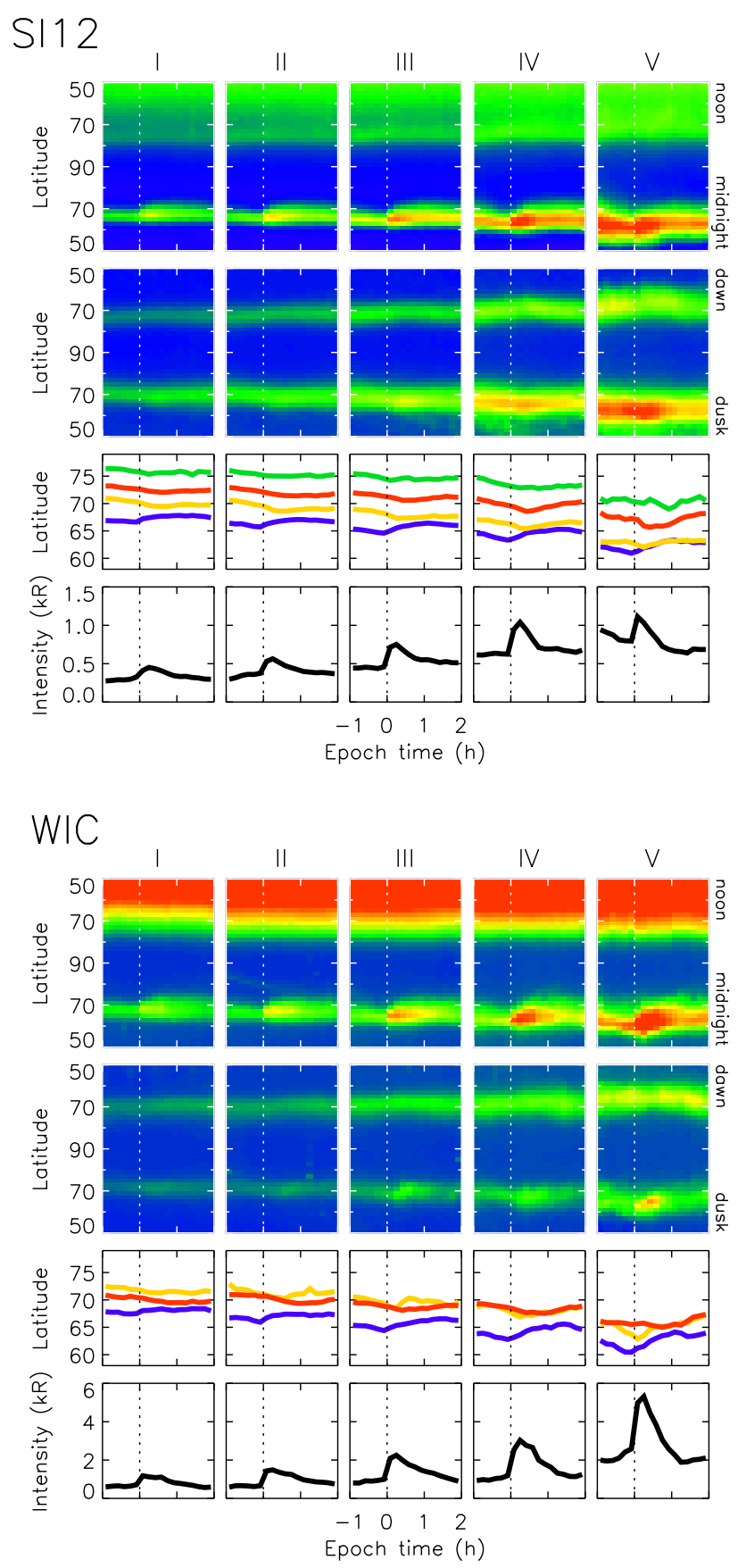

Fig. 5. The results of the superposed epoch analysis of the SI12 (upper panels) and WIC (lower panels) observations. These are presented as keograms along the noon-midnight and dawn-dusk meridians as a function of epoch time. Also indicated are the variations in latitude of the peak in intensity along the midnight (blue), noon (green), dawn (red), and dusk (yellow) meridians, and the midnight oval intensity (black).

and Gaussian auroral contribution are shown separately in Fig. 4b and d, by dashed and solid curves, respectively. The contribution of dayglow to the noon meridian background profile is clearly apparent. However, despite the fact that the noon sector oval is not very bright, it can still be discerned above the background in SI12 images; this is not the case for WIC where the dayglow obscures the auroral emission.

Figure 5 presents the full results of the superposed epoch analysis. Upper panels show the SI12 results, lower panels the WIC results. These are presented as keograms along the noon-midnight and dawn-dusk meridians. In addition, the variation in the latitude of the peak in intensity of the auroral oval $\left(\Lambda_{\max }\right)$ along the four meridians as a function of epoch time is indicated, as well as the variation in brightness of the midnight sector oval ( $\left.A_{\max }\right)$.

Several conclusions can be drawn immediately from the observations. The brightness of the auroral oval, both prior to onset and during the expansion phase, increases as the latitude of onset decreases. The SI12 oval is brighter on the dusk side and the WIC oval is brighter on the dawn side, as mentioned before. There is a dawn-dusk offset in the locations of the SI12 and WIC ovals: in the SI12 observations the dusk sector oval is located consistently 2 to 4 degrees of latitude equatorward of the dawn sector oval; in the WIC observations the dawn and dusk locations are more symmetrical. This offset is consistent with the findings of Mende et al. (2003). The increase in brightness in the midnight sector following onset, especially as observed by WIC, increases with decreasing onset latitude. Onset is accompanied by an increase in brightness along the dusk meridian for both WIC and SI12, with no appreciable increase at dawn, indicating considerable asymmetry in the substorm auroral bulge, consistent with the findings of Gjerloev et al. (2007). There is a marked decrease in the oval latitude along all meridians during the growth phase, though this is most pronounced for low latitude onsets. The midnight sector oval progresses poleward promptly following onset; the rate and duration of the poleward progression increases for decreasing onset latitude. The other meridians can display continued equatorward motion for between $20 \mathrm{~min}$ and $1 \mathrm{~h}$ after onset.

To accompany Fig. 5 we conducted superposed epoch analyses of solar wind conditions as observed by ACE (McComas et al., 1998; Smith et al., 1998; Stone et al., 1998) and lagged to the magnetopause, and geomagnetic indices, again keyed to substorm onset times and subdivided by onset latitude, for the same two-year period. These are IMF $B_{Z}$, solar wind speed $V_{S W}$, density $N_{S W}$, and dynamic pressure $P_{S W}, \mathrm{AU}, \mathrm{AL}$ and SYM-H, presented in Fig. 6 (in a similar format to Fig. 1). The main findings here are that IMF $B_{Z}$ becomes more negative prior to low latitude substorms, while $V_{S W}, N_{S W}$, and $P_{S W}$ all increase. The AL signature becomes more pronounced for the low latitude onsets. Finally, SYM-H tends to be more negative, indicating an enhanced ring current, during the low latitude onsets. These observations will be discussed further in the next section. 


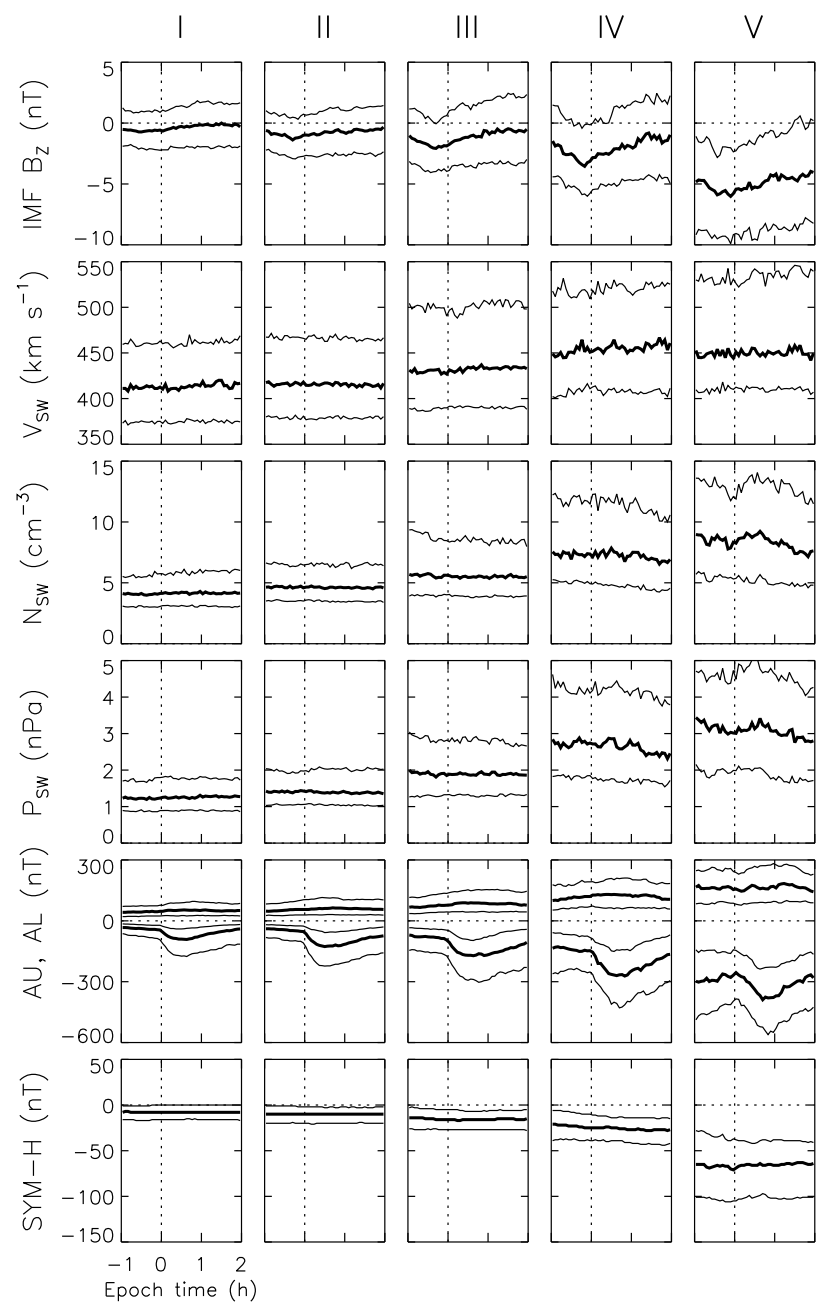

Fig. 6. Superposed epoch analyses of IMF $B_{Z}$, solar wind velocity $V_{S W}$, density $N_{S W}$, and dynamic pressure $P_{S W}$, the AU and AL indices and SYM-H for the 1993 substorms considered in our study.

\section{Discussion}

We have conducted two superposed epoch analyses of substorm characteristics, keyed to substorm expansion phase onset times. In the first we averaged measured parameters such as open magnetic flux $F_{P C}$ and nightside auroral brightness during 41 substorms. In the second we produced time-series of averaged auroral images from nearly 2000 substorms, and from these we derived the average latitude of the oval and its brightness. In both studies we subdivided our dataset by the amount of open flux in the magnetosphere prior to substorm onset, explicitly by $F_{P C}$ in the first study, and by onset latitude in the second. The conclusions to be drawn from the two studies are broadly consistent with each other. The main conclusion is that substorms are more intense when the open flux accumulated prior to onset is greater, as first suggested by Akasofu (1975) and Kamide et al. (1999).
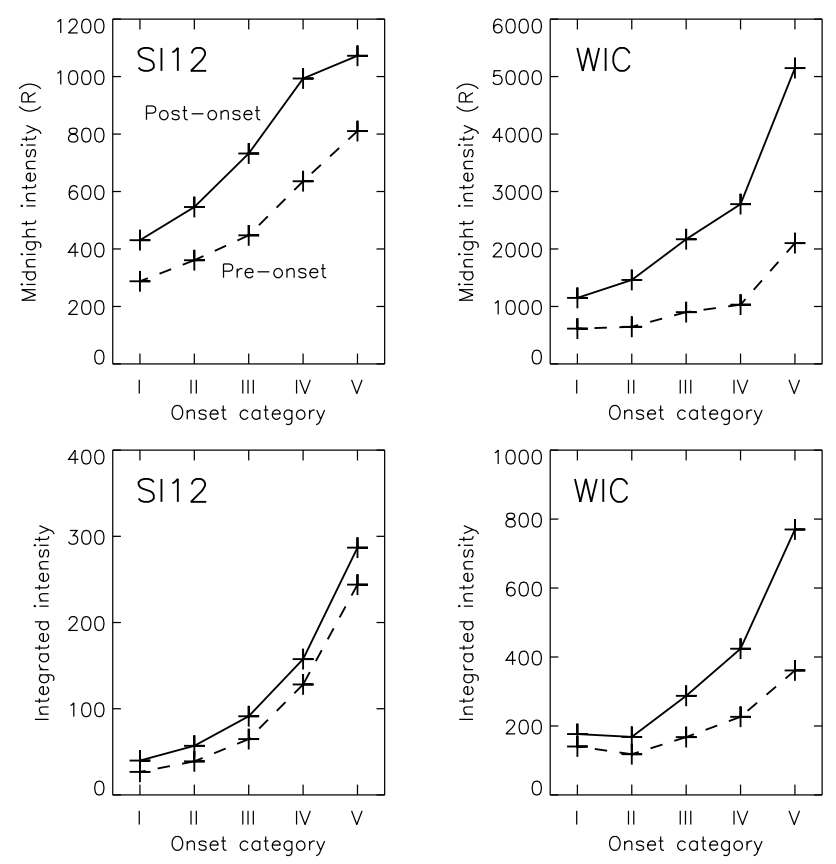

Fig. 7. The variations in pre- and post-onset brightness for the different onset latitude categories of substorm. Upper panels show the midnight intensity of the oval, whereas the lower panels show the integrated brightness of the whole oval.

With regard to our second study, we must be aware that not all auroral brightenings are substorm onsets, and it is possible that some of the events identified in the list of Frey et al. (2004) may be associated with other auroral phenomena such as pseudo-breakups or poleward boundary intensifications (PBIs), the latter described by Lyons et al. (1999) and discussed in the context of substorm identification by Rostoker (2002). True substorm onsets occur near the equatorward edge of the auroral oval, mapping close to the inner edge of the plasma sheet, deep within the closed field line region (e.g. Samson et al., 1992). By contrast, PBIs are brief, $\sim 10$ min quasi-periodic brightenings of the poleward boundary of the auroral oval, associated with activations of the plasma sheet in the more distant tail. Substorms and PBIs are hence manifestations of the "double oval" nature of the nightside auroral emissions discussed by Elphinstone et al. (1995). Frey et al. (2004) did not specifically exclude PBIs from their list, but their selection criteria (2) and (3), discussed in Sect. 3, designed to exclude pseudo-breakups, should also rule out many PBI events. For instance, criterion (3) excludes events which occur within $30 \mathrm{~min}$ of a previous auroral activation, which will catch many periodic PBIs. In addition, criterion (2) states that to be counted as substorms activations must expand up to the poleward boundary of the auroral oval and spread azimuthally for $20 \mathrm{~min}$ or more, which is not consistent with the characteristic behaviour of PBIs. Hence, we are confident that most events in our analysis are 
substorms, though it is possible that a small fraction of high latitude events, for instance in category I, may be PBIs.

Figure 7 shows the variation in emission intensity of substorms as a function of onset category. Each of the upper panels shows the brightness along the midnight meridian, the average pre-onset (dashed curves) and the maximum post-onset (solid curves), for SI12 and WIC. The lower panels show the integrated brightness of the whole auroral oval (in arbitrary units), pre- and post-onset. In the case of SI12, the midnight meridian post-onset brightness increases by almost a factor of 3 from category I to category V; for WIC this is closer to a factor of 5 . In terms of integrated brightness, the SI12 oval increases in intensity by a factor of 8; WIC by comparison increases only by a factor of 4 . These results show that the change in brightness in the WIC images is concentrated largely on the nightside in the vicinity of the auroral bulge, whereas the SII2 oval as a whole increases in brightness and such that the increase is not so concentrated into a nightside auroral bulge.

Looking at the increase in brightness from pre- to postonset within each onset category, in the case of WIC the midnight brightness and integrated brightness both increase by approximately a factor of 2 . The increase from pre- to postonset is much less pronounced in the case of SI12. The variation in SI12 pre-onset brightness with onset category can be understood in terms of pitch-angle scattering of protons in the stretched magnetic field lines of the tail. As discussed by Sergeev et al. (1983) and Blockx et al. (2005), as the nightside magnetic field becomes more tail-like, the isotropy boundary moves equatorwards and the proton aurora increase in brightness due to protons being pitch-angle scattered into the loss cone by the tight curvature of the non-dipolar field lines. Lower onset latitudes indicate a larger polar cap and hence a more tail-like field. The observations suggest that substorm onset does not significantly increase the number of precipitating protons above the level supplied by pitch angle scattering pre-onset.

The significant increase in brightness of the substorm bulge aurora with decreasing onset latitude, particularly in the electron aurora, will affect the electrodynamics of the substorm process. High conductivities in the auroral bulge lead to a stagnation in the nightside ionospheric convection, effected through increased frictional coupling between enhanced ionospheric electron and ion densities and the neutral background. Hence, high latitude substorms, being weak, will be accompanied by prompt ionospheric convection in the nightside in response to the closure of open magnetic flux (e.g. Grocott et al., 2002). Conversely, low latitude substorms will display a more sluggish ionospheric convection response, even though the closure of open flux is expected to be more pronounced.

As suggested by McPherron (1970), the substorm growth phase is the signature of accumulation of open flux by dayside reconnection. Our observations show that the increase in the size of the polar cap prior to onset is most rapid for low latitude substorms, and these are also the substorms for which IMF $B_{Z}$ is most negative and the dayside reconnection rate $\Phi_{D}$ is greatest. Similar results have been reported by, e.g. Shukhtina et al. (2005). For instance, in our first study, the level of dayside reconnection prior to onset increases from category i to category iv, from an average of $20-30 \mathrm{kV}$ to an average of $100 \mathrm{kV}$. This explains the more dramatic accumulation of open flux during the growth phase in the last category. A dayside reconnection rate of $100 \mathrm{kV}$ should produce $0.36 \mathrm{GWb}$ of open flux in one hour, whereas $30 \mathrm{kV}$ will produce just $0.11 \mathrm{GWb}$ in the same time, closely matching the observed increase in open flux during the growth phases of categories i and iv (Fig. 1).

We find that after onset the midnight sector oval expands polewards as the substorm bulge develops (Fig. 5), indicating the closure of open flux on the nightside by magnetotail reconnection (see also, Fig. 1). However, the auroral oval can continue to progress equatorwards after onset at other local times (especially in the dawn sector), indicating that open flux can still continue to accumulate if the IMF conditions are favourable, as also noted by Coumans et al. (2007). We expect that this continued equatorward motion will be more significant for low latitude onsets as the dayside reconnection rate tends to be higher at these times, but also due to the stagnation of flows in the region of the auroral bulge, as discussed above, which will impede the redistribution of open flux in the polar regions leading to a non-circular polar cap.

As discussed above, substorms are more intense the larger $F_{P C}$ at the time of onset. Why, then, does the magnetosphere sometimes allow the open flux to accumulate to large values before an intense substorm is triggered, whereas in other cases weak substorms are triggered at low levels of open flux? Milan et al. (2008) suggested that the presence of an intensified ring current introduced a significant $B_{Z}$ magnetic component into the near-tail, stabilizing it to reconnection, requiring an elevated amount of open flux in the system to trigger substorm onset. Indeed, we find in both studies reported in this paper that SYM-H is on average depressed at the times of lower latitude onsets. As substorms themselves contribute to the enhancement of the ring current, we suggest that a feedback process may be able to operate while the dayside coupling rate remains high. If the ring current becomes intensified during strong solar wind driving conditions then more open flux will accumulate prior to each substorm onset, resulting in more intense substorms, which maintain an intensified ring current.

Finally, we note that, on average, the WIC and SI12 aurora are offset along the dawn-dusk meridian with respect to each other. The WIC oval is roughly symmetrical about the noon-midnight meridian, whereas the SI12 oval is displaced towards dusk by approximately $2^{\circ}$ of latitude, such that the SI12 (WIC) oval is at lower latitude with respect to the WIC (SI12) oval in the dusk (dawn) sector. A similar offset between WIC and SI12 observations has previously been reported by Mende et al. (2003). This pattern resembles 


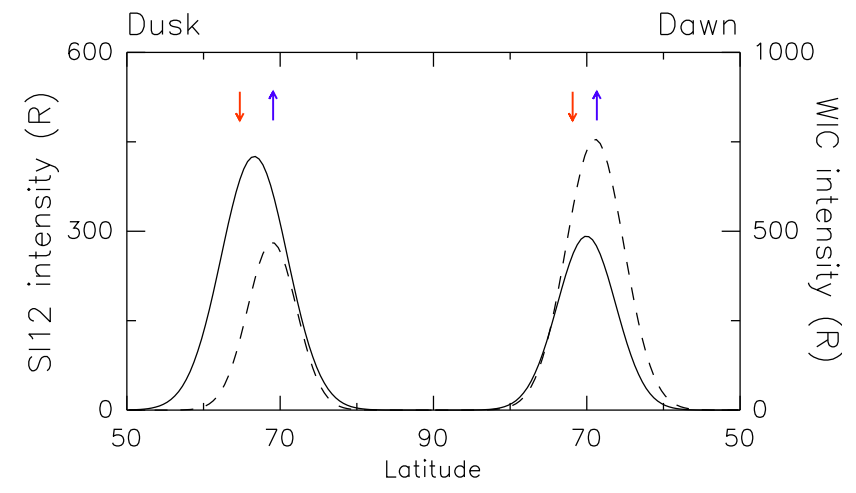

Fig. 8. The dawn-dusk meridian profiles of average auroral intensity (category IV, pre-onset) as observed by SI12 (solid curves) and WIC (dashed curves), as well as the average locations of the upward (blue arrows) and downward (red arrows) region 1 and 2 field-aligned currents identified by Iijima and Potemra (1978).

the locations of the upward and downward field aligned currents that comprise the Region 1 and 2 field aligned current (FAC) system (Iijima and Potemra, 1978), in which downward (upward) FAC is located at lower latitude with respect to downward (upward) FAC in the dusk (dawn) sector. Figure 8 shows the SI 12 and WIC intensity along the dawn-dusk meridian for category IV substorms, pre-onset, as well as the locations of the upward and downward FACs identified by Iijima and Potemra (1978). The relative locations of upward and downward FACs and WIC and SI12 aurora suggest that aurora produced by precipitating electrons are associated with the upward FAC regions and aurora produced by precipitating protons are associated with the downward FAC regions, as also discussed by Mende et al. (2003) and Boakes et al. (2008).

\section{Summary}

Substorms are more intense the greater the amount of open flux in the magnetosphere prior to onset, both in terms of the auroral brightness of the break-up aurora and the amount of open flux closed during the substorm. The auroral brightness prior to substorm onset is also greater. In the case of proton aurora this is understood in terms of pitch angle scattering in the stretched tail field, with substorm expansion phase producing only a modest increase in brightness over the preonset level. In the case of electron aurora, although the preonset brightness increases with open flux, the largest increase in brightness occurs with the onset of the expansion phase.

As substorm intensity is governed by the open flux content of the magnetosphere, it is germane to ask what controls the open flux level at which a particular substorm is triggered. The superposed epoch analyses of the solar wind conditions prior to onset suggest that the velocity and density are somewhat elevated and that IMF $B_{Z}$ is most negative prior to low latitude substorms; correspondingly the predicted dayside reconnection rate is also elevated. These indicate that the accumulation of open flux during substorm growth phase is more rapid prior to low latitude onsets. However, it seems likely that conditions internal to the magnetosphere must also stabilize the tail to substorm onset such that the open flux can grow to large values before reconnection is triggered to reclose it. The analyses presented indicate that low latitude onsets occur when the ring current is enhanced (SYM-H is depressed to low values), which will maintain a large $B_{Z}$ magnetic component in the near-Earth tail, inhibiting the onset of reconnection until the build-up of large amounts of open flux produces a very stretched topology.

The present study comprises nearly 2000 substorms. This large number will allow our dataset to be further subdivided into smaller categories and still maintain statistical significance. Hence, in future, we will be able to produce superposed epoch analyses of the auroral evolution of substorms which will investigate the response to differing onset MLT (see Fig. 2b), or different geophysical conditions of interest, for instance different orientations of IMF $B_{Y}$ or different seasons.

Acknowledgements. AG was supported by PPARC rolling grant no. PP/E000983/1. PDB was supported by a PPARC/STFC CASE award, grant no. PPA/S/C/2006/04488. CF and SMI were supported by PPARC grants PPA/S/S/2005/04156 and PPA/S/S/2005/04157, respectively. The IMAGE FUV data were supplied by the NASA Space Science Data Centre (NSSDC), and we are grateful to the PI of FUV, S. B. Mende of the University of California at Berkeley, for its use. The ACE data used in this study were accessed through CDAWeb. The authors would like to thank N. F. Ness at the Bartol Research Institute and D. J. McComas of the Southwest Research Institute, for use of the MAG and SWEPAM data, respectively.

Topical Editor I. A. Daglis thanks G. Rostoker and another anonymous referee for their help in evaluating this paper.

\section{References}

Akasofu, S.-I.: The development of the auroral substorm, Planet. Space Sci., 12, 273-282, 1964.

Akasofu, S.-I.: The roles of the north-south component of the interplanetary magnetic field on large-scale auroral dynamics observed by the DMSP satellite, Planet. Space Sci., 23, 1349-1354, 1975.

Blockx, C., Gerard, J.-C., Meurant, M., Hubert, B., and Coumans, V.: Far ultraviolet remote sensing of the isotropy boundary and magnetotail stretching, J. Geophys. Res., 110, A11215, doi:10.1029/2005JA011103, 2005.

Boakes, P. D., Milan, S. E., Abel, G. A., Freeman, M. P., Chisham, G., Hubert, B., and Sotirelis, T.: On the use of IMAGE FUV for estimating the latitude of the open/closed magnetic field line boundary in the ionosphere, Ann. Geophys., 26, 2759-2769, 2008, http://www.ann-geophys.net/26/2759/2008/.

Carbary, J. F., Sotirelis, T., Newell, P. T., and Meng, C.-I.: Auroral boundary correlations between UVI and DMSP, J. Geophys. Res., 108, 1081, doi:10.1029/2002JA009378, 2003. 
Coumans, V., Blockx, C., Gerard, J.-C., Hubert, B., and Connors, M.: Global morphology of substorm growth phases observed by the IMAGE-SI12 imager, J. Geophys. Res., 112, A11211, doi:10.1029/2007JA012329, 2007.

Cowley, S. W. H. and Lockwood, M.: Excitation and decay of solar wind-driven flows in the magnetosphere-ionosphere system, Ann. Geophys., 10, 103-115, 1992.

Dungey, J. W.: Interplanetary magnetic fields and the auroral zones, Phys. Rev. Lett., 6, 47-48, 1961.

Elphinstone, R. D., Murphree, J. S., Hearn, D. J., Cogger, L. L., Sandahl, I., Newell, P. T., Klumpar, D. M., Ohtani, S., Sauvaud, J. A., Potemra, T. A. Mursula, K., Wright, A., and Shapshak, M.: The Double Oval UV Auroral Distribution, 1. Implications for the Mapping of Auroral Arcs, J. Geophys. Res., 100, 1207512092, 1995.

Feldstein, Y. I. and Starkov, G. V.: Dynamics of auroral belt and geomagnetic disturbances, Planet. Space Sci., 15, 209-229, 1967.

Frey, H. U., Mende, S. B., Angelopoulos, V., and Donovan, E. F.: Substorm onset observations by IMAGE-FUV, J. Geophys. Res., 109, A10304, doi:10.1029/2004JA010607, 2004.

Gjerloev, J. W., Hoffman, R. A., Sigwarth, J. B., and Frank, L. A.: Statistical description of the bulge-type auroral substorm in the far ultraviolet, J. Geophys. Res., 112, A07213, doi:10.1029/2006JA012189, 2007.

Grocott, A., Cowley, S. W. H., Sigwarth, J. B., Watermann, J. F., and Yeoman, T. K.: Excitation of twin-vortex flow in the nightside high-latitude ionosphere during an isolated substorm, Ann. Geophys., 20, 1577-1601, 2002, http://www.ann-geophys.net/20/1577/2002/.

Holzworth, R. H. and Meng, C.-I.: Mathematical representation of the auroral oval, Geophys. Res. Lett., 2, 377-380, 1975.

Iijima, T. and Potemra, T. A.: Large-scale characteristics of fieldaligned currents associated with substorms, J. Geophys. Res., 83, 599-615, 1978.

Kamide, Y., Kokubun, S., Bargatze, L. F., and Frank, L. A.: The size of the polar cap as an indicator of substorm energy, Phys. Chem. Earth (C), 24, 119-127, 1999.

Kan, J. R. and Lee, L. C.: Energy coupling function and solar windmagnetosphere dynamo, Geophys. Res. Lett., 6, 577-580, 1979.

Lockwood, M. and Cowley, S. W. H.: Ionospheric convection and the substorm cycle, in: Proceedings of the International Conference on Substorms (ICS-1), 99-109, 1992.

Lyons, L. R., Nagai, T., Blanchard, G. T., Samson, J. C., Yamamoto, T., Mukai, T., Nishida, A., and Kokubun, S.: Association between Geotail plasma flows and auroral poleward boundary intensifications observed by CANOPUS photometers, J.Geophys. Res., 104, 4485-4500, 1999.

McComas, D. J., Bame, S. J., Barker, P., Feldman, W. C., Phillips, J. L., Riley, P., and Griffee, J. W.: Solar Wind Electron Proton Alpha Monitor (SWEPAM) for the Advanced Composition Explorer, Space Sci. Rev., 86, 563-612, 1998.
McPherron, R. L.: Growth phase of magnetospheric substorms, J. Geophys. Res., 75, 5592-5599, 1970.

Mende, S. B., Heetderks, H., Frey, H. U., Lampton, M., Geller, S. P., Habraken, S., Renotte, E. Jamar, C., Rochus, P., Spann, J., Fuselier, S. A., Gerard, J.-C., Gladstone, R., Murphree, S., and Cogger, L.: Far ultraviolet imaging from the IMAGE spacecraft. 1. System design, Space Sci. Rev., 91, 243-270, 2000a.

Mende, S. B., Heetderks, H., Frey, H. U., Lampton, M., Geller, S. P., Abiad, R., Siegmund, O. H. W., Tremsin, A. S., Spann, J., Dougani, H., Fuselier, S. A., Magoncelli, A. L., Bumala, M. B., Murphree, S., and Trondsen, T.: Far ultraviolet imaging from the IMAGE spacecraft. 2. Wideband FUV imaging, Space Sci. Rev., 91, 271-285, 2000b.

Mende S. B., Frey, H. U., Morsony, B. J., and Immel, T. J.: Statistical behavior of proton and electron auroras during substorms, J. Geophys. Res., 108, 1339, doi:10.1029/2002JA009751, 2003.

Milan, S. E., Lester, M., Cowley, S. W. H., Oksavik, K., Brittnacher, M., Greenwald, R. A., Sofko, G., and Villain, J.-P.: Variations in the polar cap area during two substorm cycles, Ann. Geophys., 21, 1121-1140, 2003, http://www.ann-geophys.net/21/1121/2003/.

Milan, S. E., Provan, G., and Hubert, B.: Magnetic flux transport in the Dungey cycle: A survey of dayside and nightside reconnection rates, J. Geophys. Res., 112, A01209, doi:10.1029/2006JA011642, 2007.

Milan, S. E., Boakes, P. D., and Hubert, B.: Response of the expanding/contracting polar cap to weak and strong solar wind driving: implications for substorm onset, J. Geophys. Res., 113, A09215, doi:10.1029/2008JA013340, 2008.

Rostoker, G.: Identification of substorm expansive phase onsets, J. Geophys. Res., 107, 1137, doi:10.1029/2001JA003504, 2002.

Samson, J. C., Lyons, L. R., Newell, P. T., Creutzberg, F., and Xu, B.: Proron aurora and substorm intensifications, Geophys. Res. Lett., 19, 2167-2170, 1992.

Sergeev, V. A., Sazhina, E. M., and Tsyganenko, N. A.: Pitch-angle scattering of energetic protons in the magnetotail current sheet as the dominant source of their isotropic precipitation into the nightside ionosphere, Planet. Space Sci., 31, 1147-1155, 1983.

Shukhtina, M. A., Dmitrieva, N. P., Popova, N. G., Sergeev, V. A., Yahnin, A. G., and Despirak, I. V.: Observational evidence of the loading-unloading substorm scheme, Geophys. Res. Lett., 32, L17107, doi:10.1029/2005GL023779, 2005.

Siscoe, G. L. and Huang, T. S.: Polar cap inflation and deflation, J. Geophys. Res., 90, 543-547, 1985.

Smith, C. W., L'Heureux, J., Ness, N. F., Acuña, M. H., Burlaga, L. F., and Scheifele, J.: The ACE Magnetic Field Experiment, Space Sci. Rev., 86, 613-632, 1998.

Stone, E. C., Frandsen, A. M., Mewaldt, R. A., Christian, E. R., Marglies, D., Ormes, J. F., and Snow, F.: The Advanced Composition Explorer, Space Sci. Rev., 86, 1-22, 1998. 